\title{
Retraction: Ethanol Mediates Cell Cycle Arrest and Apoptosis in SK-N-SH Neuroblastoma Cells
}

Maria Lee', Byoung-Joon Song ${ }^{2}$, Yongil Kwon ${ }^{3}$

${ }^{1}$ Department of Obstetrics and Gynecology, Seoul National University College of Medicine, Seoul National University Hospital, Seoul, Korea, ${ }^{2}$ Section of Molecular Pharmacology and Toxicology, Laboratory of Membrane Biochemistry and Biophysics, National Institute on Alcohol Abuse and Alcoholism, Bethesda, USA, ${ }^{3}$ Department of Obstetrics and Gynecology, Kangdong Sacred Heart Hospital, Hallym University, Seoul, Korea

J Cancer Prev 2014;19:39-46

http://dx.doi.org/10.15430/JCP.2014.19.1.39

This article has been retracted following a review by the Editorial Board. It has come to light that there is a significant overlap between the article and one that had already appeared in Neural Regeneration Research 2013:8(20):1853-1862 (doi: 10.3969/j.issn.1673-5374.2013.20.004). 